\title{
Modeling nanoindentation of virus shells: Do substrate adhesion and geometry matter?
}

\author{
Arthur Bousquet ${ }^{* 1}$, Bogdan Dragnea ${ }^{2}$, Manel Tayachi ${ }^{1}$, and Roger Temam \\ $\dagger 1$ \\ ${ }^{1}$ Department of Mathematics and Institute for Scientific Computing and Applied Mathematics, \\ Indiana University, Rawles Hall, 831 East 3rd St Bloomington, IN 47405-5701, USA \\ ${ }^{2}$ Department of Chemistry, Indiana University, 800 E. Kirkwood Ave. Bloomington, IN \\ 47405-7102, USA.
}

December 27, 2015

\begin{abstract}
This article investigates the deformation of a shell representing a virus in the context of slow evolution linear elasticity and in contact of different substrates. In order to understand the influence of the adhesion forces in the deformation of the virus, we compare the virus binding in contact of a flat substrate with and without the adhesion forces derived from the Lennard-Jones potential. Finally, we study the influence of the geometry of the substrate by considering a support made of two circles. The numerical simulations use a finite elements discretization.
\end{abstract}

Keywords: virus binding, linear elasticity, unilateral contact, virus interaction with substrate, finite elements.

\section{Introduction}

Recent past has witnessed significant improvements in techniques for the measurement of forces at nanoscale [2] and an increased interest in the physical phenomena underlying the behavior of viruses as many-body molecular systems $[18,16]$. As a consequence there is an increased demand on theoretical models for problems relating to the interaction of a virus with its environment.

${ }^{*}$ Present address: Penn State University Mathematics Department, University Park, State College, PA 16802

${ }^{\dagger}$ Electronic address: temam@indiana.edu; Corresponding author 
For example, viruses have to bind and cross the plasma membrane. It is well established that instances of multivalent virus binding to the host plasma membrane trigger signaling pathways in the cell which lead to virus endocytosis [15]. Similarly, binding to the endosomal membrane triggers in certain viruses profound conformational changes [25]. How is virus mechanics involved in translating the binding events into a chemical transformation cascade? This type of problem is important because virus deformation could be an integral part of various stages of the virus life cycle.

Numerous experimental studies of virus deformation using the mechanical probe of an atomic force microscope have already provided a wealth of information on the strength of a virus shell and on the dynamics of deformation under directional load; see e.g. [3, 8, 9, 10, 17, 20, 23, 24]. Theoretical treatments followed, from simple thin elastic shell approximations, through finite-element numerical simulations of continuum models, to coarse-grained molecular dynamics $[19,1,5]$. In all these models, the interaction between the substrate and the virus during compression by a mechanical probe is reduced to a rough contact and a hardwall potential, i.e. the particle cannot slip and only very short-range repulsive interactions occur between the surface and the elements of the virus model.

However, in reality, viruses have to bind to the substrate surface to be immobilized for atomic force microscope (AFM) imaging. Attractive forces must exist between substrate and the virus shell. As the shell is pressed against the substrate by the AFM probe, adhesion increases. The question we address here is how might these attractive forces might influence the force-displacement curve and the measurement of an elastic constant for the virus particle?

The mathematical model that we will use is based on linear elasticity theory with unilateral contact, first developed from a mathematical point of view by G. Fichera [7], J.-L. Lions and G. Stampacchia [14], and G. Duvaut and J.-L. Lions [6] and by N. Kikuchi and J. T. Oden from a numerical point of view [12]. We consider in the sequel an elastic shell representing the virus with initial shape $\Omega^{0}$ and subjected to forces $\mathcal{F}$, combination of an imposed force $\mathbf{F}$ and substrate adhesion forces $\mathbf{f}_{a d h}$ derived from the Lennard-Joness potential [11]. The shell is subjected to unilateral contact with a rigid support. We assume that the force F is progressively applied by small increments $\frac{\mathbf{F}}{N}$, where $N \in \mathbf{N}^{*}$, like in a slow evolution and that the body is subjected to a succession of equilibrium states and that it remains elastic all along. In particular we assume that the passage from the equilibrium under the force $\frac{n \mathbf{F}}{N}$, where $0 \leq n<N$, to the equilibrium under the force $\frac{(n+1) \mathbf{F}}{N}$ pertains to linear elasticity. Note that the "linear elasticity" behavior applies to the constitutive law only since the problem is nonlinear in any case due to the unilateral forces as described below.

This paper is organized as follows: in Section 2, we start by writing the set of inequations of linear elasticity modeling the deformation of a virus subject to an exterior force and in contact with a solid support. In a first approximation this rigid surface could represent the cell surface. Then we give some classical theoretical results regarding the existence and uniqueness of the solution for such a model. To avoid remeshing the domain at each increment of force, we chose to do all the calculations in the initial domain $\Omega^{0}$ representing the 
initial shape of the virus, thus implementing in fact an Euler-Lagrange representation. We proceed in Section 2.3 to a change of variables in order to express the variational formulation in terms of the variables in $\Omega^{0}$. In Section 3 we introduce the incremental algorithm and the discretization of the variational formulation using a finite elements method. Finally, Section 4 is dedicated to numerical simulations. We discuss some test cases representing the deformation of a virus in contact with a flat support and in contact with two circles with and without the adhesion force. The force-deformation figure (Figure 8), shows the highly non-linear behavior of the virus, even if we used a linear elasticity constitutive law. See [9] for discussions on the utilization of several different non-linear constitutive laws. We ourselves intend in future works to study the effect of using some nonlinear constitutive laws (nonlinear elasticity, plasticity possibly) and to compare the results to the current study.

\section{Mathematical modeling}

To model the virus deformation, the procedure is as follows: we start from a state $\Omega^{n}$ (which could be $\Omega^{0}$ ) corresponding to a force $\frac{n F}{N}$. Then we apply a force $\frac{(n+1) \mathbf{F}}{N}$. For practical (computational) reasons, we chose to make the numerical calculations in the undeformed state $\Omega^{0}$. We call $\varphi^{n}$ the application mapping $\Omega^{0}$ onto $\Omega^{n}=\varphi^{n}\left(\Omega^{0}\right)$. The incremental force treated in the realm of linear elasticity produces a "small" displacement $\mathbf{u}^{n}$. Then $\Omega^{n+1}=\left\{\mathbf{x}+\mathbf{u}^{n}(\mathbf{x}), \mathbf{x} \in \Omega^{n}\right\}$ and we call $\varphi^{n+1}$ the application mapping $\Omega^{0}$ onto $\Omega^{n+1}$.

In summary we start with $\Omega^{0}, \varphi^{0}=I d$ (Identity). When $\Omega^{n}$ and $\varphi^{n}$ are known we compute $\mathbf{u}^{n}$ (the displacement starting from $\Omega^{n}$ ), and $\varphi^{n+1}$ by setting

$$
\begin{aligned}
& \varphi^{n}\left(\mathrm{x}^{0}\right)=\mathrm{x}^{n}, \quad \mathbf{x}^{0} \in \Omega^{0}, \quad \mathbf{x}^{n} \in \Omega^{n}, \\
& \varphi^{n+1}\left(\mathrm{x}^{0}\right)=\mathrm{x}^{n}+\mathbf{u}^{n}\left(\mathrm{x}^{n}\right) .
\end{aligned}
$$

At the end we obtain $\Omega^{N}=\Omega^{\varphi}$ and $\varphi^{N}=\varphi$ the final state and the final mapping $\Omega^{\varphi}=\varphi\left(\Omega^{0}\right)$ with displacement $\mathbf{u}\left(\mathrm{x}^{0}\right)=\varphi\left(\mathrm{x}^{0}\right)-\mathrm{x}^{0}$.

\subsection{Equations}

In order to write the model describing the deformation of the virus, we will first give some definitions of the physical parameters and quantities involved.

The initial domain is

$$
\Omega^{0}=\left\{r_{1} \leq \sqrt{x^{2}+y^{2}} \leq r_{2}\right\}
$$

where $r_{1}=10 \mathrm{~nm}$ and $r_{2}=15 \mathrm{~nm}$, although, obviously, other geometrical domains can be considered. The inner and outer boundaries of $\Omega$ are denoted by $\Gamma_{1}$ and $\Gamma_{2}$.

In the case of small deformations and displacements, the equation of the equilibrium of a deformable body subjected to a force $\mathbf{f}$ is given by:

$$
\operatorname{div}(\boldsymbol{\sigma}(\mathbf{u}))+\mathbf{f}=0
$$


where $\mathbf{u}=\left(\begin{array}{l}u_{1}(x, y) \\ u_{2}(x, y)\end{array}\right)$, is the displacement and $\boldsymbol{\sigma}=2 \mu \boldsymbol{\varepsilon}+\lambda \operatorname{Tr}(\boldsymbol{\varepsilon}) \boldsymbol{I} \boldsymbol{d}$ is the Cauchy stress tensor defined using the linear strain tensor

$$
\varepsilon_{i, j}=\frac{1}{2}\left(u_{i, j}+u_{j, i}\right)
$$

where $u_{i, j}=\frac{\partial u_{i}}{\partial x_{j}}$

The physical parameters $\mu$ and $\lambda$ are the Lamé coefficients and are defined by:

$$
\mu=\frac{E}{2(1+\nu)} \mathrm{nN} / \mathrm{nm}
$$

and

$$
\lambda=\frac{E \nu}{(1-2 \nu)(1+\nu)} \mathrm{nN} / \mathrm{nm}
$$

where

- $E=0.5$

- $\nu=0.35$ (Poisson's ratio)

As described below, at each iteration $n$, when $\Omega^{n}$ and $\varphi^{n}$ are known we suppose that we apply to the virus the force $\mathbf{F}^{n+1}$. We divide the outer boundary $\Gamma_{2}^{n}$ of $\Omega^{n}$ in three non intersecting parts, $\Gamma_{D}^{n}, \Gamma_{P}^{n}$ and $\Gamma_{C}^{n}$ such that $\Gamma_{2}^{n}=\Gamma_{D}^{n} \cup \Gamma_{P}^{n} \cup \Gamma_{C}^{n} ; \Gamma_{D}^{n}$ is the part of $\Gamma_{2}$ where the displacement is imposed, $\Gamma_{P}^{n}$ is the part of $\Gamma_{2}$ in contact with the punch and $\Gamma_{C}^{n}$ is the part of $\Gamma_{2}$ in contact with the support. Finally, we suppose also the contact to be rigid; therefore the virus cannot penetrate the support, and we suppose that there is no friction with the contact. One can then propose the following set of equations and inequations, see e.g.[6], [12] and [22]:

$$
\left\{\begin{array}{r}
\boldsymbol{\sigma}_{i j, j}^{n}+\mathbf{f}_{a d h, i}^{n}=0, \text { in } \Omega^{n} \\
\mathbf{u}^{n}=\mathbf{u}_{0}, \text { on } \Gamma_{D}^{n}, \\
\boldsymbol{\sigma}^{n} \mathbf{n}=0, \text { on } \Gamma_{1}^{n} \\
\boldsymbol{\sigma}^{n} \mathbf{n}=\mathbf{F}^{n+1}, \text { on } \Gamma_{C}^{n} \\
\boldsymbol{\sigma}^{n} \mathbf{n}=0, \text { on } \Gamma_{D}^{n} \cup \Gamma_{P}^{n}, \\
\mathbf{u}_{N}^{n}-g^{n}=\mathbf{u}_{i}^{n} \mathbf{n}_{i}-g^{n} \leq 0, \text { on } \Gamma_{C}^{n} \\
\boldsymbol{\sigma}_{N}^{n}=\sigma_{i j}^{n} \mathbf{n}_{i} \mathbf{n}_{j} \leq 0, \text { on } \Gamma_{C}^{n} \\
\left(\boldsymbol{\sigma}_{T}^{n}\right)_{i}=\boldsymbol{\sigma}_{i j}^{n} \mathbf{n}_{j}-\boldsymbol{\sigma}_{N}^{n} n_{i}=0, \text { on } \Gamma_{C}^{n} \\
\boldsymbol{\sigma}_{N}^{n}\left(u_{N}-g^{n}\right)=0, \text { on } \Gamma_{C}^{n}
\end{array}\right.
$$

Here the vector $\sigma^{n} \mathbf{n}$ is decomposed into the sum of its tangential and normal components:

$$
\boldsymbol{\sigma}^{n} \mathbf{n}=\boldsymbol{\sigma}_{T}^{n}+\boldsymbol{\sigma}_{N}^{n} \mathbf{n}
$$


with $\mathbf{n}$ the outward unit normal to the virus and $\boldsymbol{\sigma}_{N}^{n}=\sigma^{n} \mathbf{n} \cdot \mathbf{n}$. We impose a Dirichlet boundary condition on $\Gamma_{D}^{n}$ and without lack of generality we will suppose $\mathbf{u}_{0}=\mathbf{0}$. The force $\mathbf{F}^{n+1}=-\frac{(n+1) F}{N} \mathbf{n}$, where $F$ is a constant, is applied on $\Gamma_{P}^{n}$ and $g^{n}$ is the distance between the zone of contact $\Gamma_{C}^{n}$ and the rigid support. In our study we will suppose that $g^{n}=0$. When $g_{n}>0$, the virus is not in reality in geometrical contact with the support along $\Gamma_{C}^{n}$, and this is consistent with the biological context (see below the definition of $\mathbf{f}_{a d h}^{n}$ ). We denote by $\mathbf{f}_{a d h}^{n}$ the adhesion force from the contact that we define later in Section 2.3.3. We will now study briefly the system (2).

\subsection{Variational formulation}

In order to write the variational formulation of the system (2) we introduce the following function space and set:

$$
K^{n}=\left\{\mathbf{v}^{n} \in \mathbf{V}^{n}: \mathbf{v}_{N}^{n}=\mathbf{v}^{n} \cdot \mathbf{n} \leq 0 \text { on } \Gamma_{C}^{n}\right\}
$$

where

$$
\mathbf{V}^{n}=\left\{\mathbf{v}^{n} \in H^{1}\left(\Omega^{n}\right)^{2}: \mathbf{v}^{n}=0 \text { on } \Gamma_{D}^{n}\right\} .
$$

Now by multiplying the first equation of the system (2) by $\mathbf{v}^{n}-\mathbf{u}^{n}$ where $\mathbf{v}^{n} \in K^{n}$ and by integrating in $\Omega^{n}$ and in view of the Green formula and of the boundary conditions, we obtain:

$$
\begin{aligned}
& -\int_{\Omega^{n}} 2 \mu \varepsilon_{i j}^{n}\left(\mathbf{u}^{n}\right) \varepsilon_{i j}^{n}\left(\mathbf{v}^{n}-\mathbf{u}^{n}\right) d \mathbf{x}^{n}-\int_{\Omega^{n}} \lambda \varepsilon_{k k}^{n}\left(\mathbf{u}^{n}\right) \varepsilon_{i i}^{n}\left(\mathbf{v}^{n}-\mathbf{u}^{n}\right) d \mathbf{x}^{n} \\
& +\int_{\partial \Omega^{n}} \sigma_{i j}^{n} n_{j}\left(v^{n}-u^{n}\right)_{i} d \sigma^{n}+\int_{\Omega^{n}} f_{a d h, i}^{n}\left(v^{n}-u^{n}\right)_{i} d \mathbf{x}^{n} \leq 0,
\end{aligned}
$$

where the Einstein convention of summation of repeated indices has been used. Using the boundary condition $(2 \mathrm{~d})$, we see that

$$
\int_{\partial \Omega^{n}} \sigma_{i j}^{n} n_{j}\left(v_{i}^{n}-u_{i}^{n}\right) d \sigma^{n}=\int_{\Gamma_{P}^{n}} \mathbf{F}^{n+1} \cdot\left(\mathbf{v}^{n}-\mathbf{u}^{n}\right) d \sigma^{n}
$$

Therefore by defining

$$
\begin{aligned}
& \mathbf{a}^{n}\left(\mathbf{u}^{n}, \mathbf{v}^{n}\right)=\int_{\Omega^{n}} 2 \mu \varepsilon_{i j}^{n}\left(\mathbf{u}^{n}\right) \varepsilon_{i j}^{n}\left(\mathbf{v}^{n}\right) d \mathbf{x}^{n}+\int_{\Omega^{n}} \lambda \varepsilon_{k k}^{n}\left(\mathbf{u}^{n}\right) \varepsilon_{i i}^{n}\left(\mathbf{v}^{n}\right) d \mathbf{x}^{n}, \\
& \mathbf{l}^{n}\left(\mathbf{v}^{n}\right)=\int_{\Gamma_{P}^{n}} \mathbf{F}^{n+1} \cdot \mathbf{v}^{n} d \sigma^{n}+\int_{\Omega^{n}} \mathbf{f}_{a d h}^{n} \cdot \mathbf{v}^{n} d \mathbf{x}^{n},
\end{aligned}
$$

the inequality (3) now reads:

$$
\mathbf{a}^{n}\left(\mathbf{u}^{n}, \mathbf{v}^{n}-\mathbf{u}^{n}\right) \geq \mathbf{l}^{n}\left(\mathbf{v}^{n}-u^{n}\right), \quad \forall \mathbf{v}^{n} \in K^{n} .
$$

Then we have the following result: 
Theorem 1 If $\mathbf{f}_{\text {adh }}^{n} \in\left(L^{2}\left(\Omega^{n}\right)\right)^{2}$ and $\mathbf{F}^{n+1} \in\left(L^{2}\left(\Gamma_{p}^{n}\right)\right)^{2}$ are given and if meas $\left(\Gamma_{D}^{n}\right) \neq 0$ then the system (2) is well-posed and has a unique solution $\mathbf{u}^{n} \in K^{n}$ satisfying the variational inequality (5). Moreover $\mathbf{u}^{n}$ is solution of the following minimization problem:

$$
\min _{\mathbf{v} \in K^{n}} \mathcal{J}(\mathbf{v})
$$

where

$$
\mathcal{J}(\mathbf{v})=\frac{1}{2} \mathbf{a}^{n}\left(\mathbf{v}^{n}, \mathbf{v}^{n}\right)-\mathbf{l}^{n}\left(\mathbf{v}^{n}\right)
$$

If meas $\left(\Gamma_{D}^{n}\right)=0$, then $\mathbf{u}^{n}$ is unique up to a rotation.

Sketch of the proof: We will give here the outlines of the proof for the convenience of the reader. More details can be found in [12] and [6].

First we will prove the formal equivalence of the weak formulation (i.e (5)) and the strong solution (i.e (2a)-(2i)). More precisely we will prove that a regular solution of the variational inequality (5) is also a solution of the system (2). Suppose that the inequality (5) holds for a function $\mathbf{u}^{n} \in K^{n}$ and let us prove that $\mathbf{u}^{n}$ is then a solution of the system (2). Let us define:

$$
\mathcal{D}\left(\Omega^{n}\right)=\left\{\boldsymbol{\Phi} \in C^{\infty}\left(\Omega^{n}\right), \boldsymbol{\Phi} \text { has a compact support in } \Omega^{n}\right\}
$$

Then $\mathbf{v}^{n}=\mathbf{u}^{n} \pm \boldsymbol{\Phi}$, where $\boldsymbol{\Phi} \in \mathcal{D}\left(\Omega^{n}\right)$, belongs to $K^{n}$ and for such a $\mathbf{v}^{n}$, the inequality (5) reads:

$$
\pm \int_{\Omega^{n}} 2 \mu \varepsilon_{i j}^{n}\left(\mathbf{u}^{n}\right) \varepsilon_{i j}(\boldsymbol{\Phi}) d \mathbf{x}^{n} \pm \int_{\Omega^{n}} \lambda \varepsilon_{k k}^{n}\left(\mathbf{u}^{n}\right) \varepsilon_{i i}^{n}(\boldsymbol{\Phi}) d \mathbf{x}^{n} \pm \int_{\Omega^{n}} \mathbf{f}_{a d h}^{n} \cdot \boldsymbol{\Phi} d \mathbf{x}^{n} \geq 0
$$

which implies:

$$
\sigma_{i j, j}^{n}+f_{a d h, i}^{n}=0 \text { in } \Omega^{n} .
$$

Using (6) and the Green formula, the inequality (5) becomes: For all $\mathbf{v}^{n} \in K^{n}$ :

$$
\int_{\Gamma_{P}^{n} \cup \Gamma_{C}^{n} \cup \Gamma_{1}^{n}} \boldsymbol{\sigma}_{i j}^{n} \mathbf{n}_{j}\left(v_{i}^{n}-u_{i}^{n}\right) d \sigma^{n} \geq \int_{\Gamma_{P}^{n}} \mathbf{F}^{n+1} \cdot\left(\mathbf{v}^{n}-\mathbf{u}^{n}\right) d \sigma^{n} .
$$

By choosing $\boldsymbol{\Phi} \in V^{n}$ such that $\boldsymbol{\Phi}=0$ on $\Gamma_{C}^{n} \cup \Gamma_{1}^{n}$ and $\mathbf{v}^{n}=\mathbf{u}^{n} \pm \boldsymbol{\Phi}$ we can deduce that:

$$
\boldsymbol{\sigma} \mathbf{n}=\mathbf{F}^{n+1} \quad \text { on } \Gamma_{P}^{n}
$$

and by using a similar argument we can show that:

$$
\boldsymbol{\sigma} \mathbf{n}=0 \quad \text { on } \Gamma_{1}^{n}
$$

The inequality (7) can be now simplified using these two last equations and we obtain:

$$
\int_{\Gamma_{C}^{n}} \sigma_{i j}^{n} n_{j}\left(v_{i}^{n}-u_{i}^{n}\right) d \sigma^{n} \geq 0
$$


Using the decomposition of the vector $\boldsymbol{\sigma}^{n} \mathbf{n}$ as the sum of its tangential and normal components; $\boldsymbol{\sigma}^{n} \mathbf{n}=\boldsymbol{\sigma}_{N}^{n} \mathbf{n}+\boldsymbol{\sigma}_{T}^{n}$ and by choosing $\mathbf{v}^{n}=\mathbf{u}^{n}+\boldsymbol{\Phi}$ where $\boldsymbol{\Phi} \in\left\{\boldsymbol{\Phi} \in V^{n}: \Phi_{N}=\boldsymbol{\Phi} \cdot \mathbf{n}=0\right\}$, we obtain:

$$
\int_{\Gamma_{C}^{n}} \sigma_{i j}^{n} n_{j}\left(v_{i}^{n}-u_{i}^{n}\right) d \sigma^{n}=\int_{\Gamma_{C}^{n}}\left(\boldsymbol{\sigma}_{N}^{n}\right)(\mathbf{n} \cdot \Phi) d \sigma^{n}+\int_{\Gamma_{C}^{n}} \boldsymbol{\sigma}_{T}^{n} \cdot \boldsymbol{\Phi} d \sigma^{n} .
$$

So we can deduce that $\int_{\Gamma_{C}^{n}} \boldsymbol{\sigma}_{T}^{n} \cdot \boldsymbol{\Phi} d \sigma^{n} \geq 0$ for all $\boldsymbol{\Phi}$ such that $\boldsymbol{\Phi} \cdot \mathbf{n}=0$ and by doing the same calculations with $\boldsymbol{- \Phi}$ we conclude that:

$$
\boldsymbol{\sigma}_{T}=0 \quad \text { on } \Gamma_{C}^{n}
$$

Finally, let $\boldsymbol{\Phi} \in V^{n}$ be such that $\Phi_{N}=\boldsymbol{\Phi} \cdot \mathbf{n} \leq 0$ on $\Gamma_{C}^{n}$; then we have $\mathbf{v}^{n}=\mathbf{u}^{n}+\Phi \in K^{n}$ and we obtain:

$$
\int_{\Gamma_{C}^{n}}\left(\boldsymbol{\sigma}_{N}^{n}\right)(\mathbf{n} \cdot \Phi) d \sigma^{n} \geq 0
$$

This implies that:

$$
\boldsymbol{\sigma}_{N}^{n} \leq 0 \quad \text { on } \Gamma_{C}^{n}
$$

which finishes the proof of the formal equivalence between the strong solution and the weak solution.

Now to prove the existence and uniqueness of a solution of the variational inequality (5), we will use the following theorem (Lions-Stampacchia [14]) and corollary (Stampacchia [21]).

Theorem 2 (Lions-Stampacchia [14])) Let $V$ be a Hilbert space and $K \in V$ a nonempty, closed, convex subset of $V$.

Let $A: V \rightarrow V^{\prime}$, where $V^{\prime}$ denotes the dual of the space $V$, be a continuous and coercive linear operator, that is there exist $k, \alpha>0$ such that:

$$
\begin{gathered}
\|A u-A v\| \leq k\|u-v\|, \quad \text { for all } \quad u, v \in K, \\
\langle A u-A v, u-v\rangle \geq \alpha\|u-v\|^{2}, \quad \text { for all } u, v \in K .
\end{gathered}
$$

Then for each $L \in V^{\prime}$, there exists a unique solution $u \in K$ of the variational inequality

$$
\langle A u-L, v-u\rangle, \quad \text { for all } v \in K
$$

As a consequence we have the following corollary (Stampacchia [21]):

Corollary 1 Let $V$ denote a real Hilbert space and $K \subset V$ a nonempty, closed, convex subset of $V$. Assume $a($,$) is a continuous, coercive bilinear form on V$ and $L \in V^{\prime}$. Then there exists a unique solution $u \in K$ of the variational inequality

$$
a(u, v-u) \geq\langle L, v-u\rangle, \quad v \in K
$$


As a next step we need to prove that $K^{n}$ is a nonempty closed and convex set of $V^{n}$. In fact $K^{n}$ is nonempty because it contains the null displacement. Let us consider $\mathbf{u}^{n}$ and $\mathbf{v}^{n} \in K^{n}$ and $0<\alpha<1$. We see that $\alpha \mathbf{u}_{N}^{n} \leq 0$ on $\Gamma_{C}^{n}$ and $(1-\alpha) \mathbf{v}_{N}^{n} \leq 0$ on $\Gamma_{C}^{n}$ and we can deduce that $\alpha \mathbf{u}_{N}^{n}+(1-\alpha) \mathbf{v}_{N}^{n} \leq 0$ on $\Gamma_{C}^{n}$ and thus $K^{n}$ is convex. To finish we prove that $K^{n}$ is closed. Let $\left(\mathbf{u}_{k}^{n}\right)_{k \in \mathbb{N}} \in K^{n}$ and $\left(\mathbf{u}_{k}^{n}\right) \rightarrow \mathbf{u}^{n}$ in $V^{n}$ which implies $\mathbf{u}_{k}^{n} \cdot \mathbf{n} \rightarrow \mathbf{u}^{n} \cdot \mathbf{n}$ in $L^{2}\left(\partial \Omega^{n}\right)$ by continuity of the trace. To finish this step one can use that if a sequence converges in $L^{2}\left(\partial \Omega^{n}\right)$ then one can extract a subsequence converging almost everywhere. As $u_{k}^{n} \cdot \mathbf{n} \leq 0$ on $\Gamma_{C}^{n}$ one can conclude that $\mathbf{u}^{n} \cdot \mathbf{n} \leq 0$ on $\Gamma_{C}^{n}$ and therefore the limit is in $K^{n}$.

Now to conclude, we need to prove that $a^{n}($,$) is continuous and coercive. The continuity is$ straightforward and to prove the coercivity we need to assume that the tensor of elasticity E defined by $E_{i j k l}=\mu\left(I_{i k} I_{j l}+I_{i l} I_{j k}\right)+\lambda I_{i j} I_{k l}$ is symmetric, it means $E_{i j k l}=E_{j i k l}=E_{i j l k}=$ $E_{k l i j, 1 \leq i, j, k, l \leq 2}$, and elliptic. This last assumption means that there exists a constant $m>0$ such that for all symmetric tensor $\boldsymbol{\epsilon}$ the following inequality holds:

$$
E_{i j k l} \epsilon_{i j} \epsilon_{k l} \geq m \epsilon_{i j} \epsilon_{k l} \text {. }
$$

Finally by using the Korn inequality we can deduce the coercivity of $a^{n}($, ) which finishes the proof of Theorem 1 .

Remark 1 For the numerical simulations we will transform the variational inequality (5) into a variational equation using the following observation (see [6]):

If $\mathbf{w}^{n} \in K_{0}^{n}=\left\{\mathbf{v} \in K, \quad \mathbf{v}^{n}=0\right.$ on $\left.\Gamma_{C}^{n}\right\}$ we can replace $\mathbf{v}^{n}$ by $\mathbf{u}^{n} \pm \mathbf{w}^{n} \in K^{n}$ and we find

$$
\begin{aligned}
& -\int_{\Omega^{n}} 2 \mu \varepsilon_{i j}^{n}\left(\mathbf{u}^{n}\right) \varepsilon_{i j}\left(\mathbf{w}^{n}\right) d \mathbf{x}^{n}-\int_{\Omega^{n}} \lambda \varepsilon_{k k}^{n}\left(\mathbf{u}^{n}\right) \varepsilon_{i i}^{n}\left(\mathbf{w}^{n}\right) d \mathbf{x}^{n} \\
& +\int_{\partial \Omega^{n}} \sigma_{i j}^{n} n_{j} w_{i}^{n} d \mathbf{x}^{n}+\int_{\Omega^{n}} f_{a d h, i}^{n} w_{i}^{n} d \mathbf{x}^{n}=0, \quad \forall \mathbf{w}^{n} \in K_{0}^{n} .
\end{aligned}
$$

Changing notations we obtain:

$$
\begin{aligned}
& -\int_{\Omega^{n}} 2 \mu \varepsilon_{i j}^{n}\left(\mathbf{u}^{n}\right) \varepsilon_{i j}\left(\mathbf{v}^{n}\right) d \mathbf{x}^{n}-\int_{\Omega^{n}} \lambda \varepsilon_{k k}^{n}\left(\mathbf{u}^{n}\right) \varepsilon_{i i}^{n}\left(\mathbf{v}^{n}\right) d \mathbf{x}^{n} \\
& +\int_{\partial \Omega^{n}} \sigma_{i j}^{n} n_{j} v_{i}^{n} d \mathbf{x}^{n}+\int_{\Omega^{n}} f_{a d h, i}^{n} v_{i}^{n} d \mathbf{x}^{n}=0, \quad \forall \mathbf{v}^{n} \in K_{0}^{n} .
\end{aligned}
$$

The equation (9) says that $\mathbf{u}^{n} \in K^{n}$ and

$$
\mathbf{a}^{n}\left(\mathbf{u}^{n}, \mathbf{v}^{n}\right)=\mathbf{l}^{n}\left(\mathbf{v}^{n}\right), \quad \forall \mathbf{v}^{n} \in K_{0}^{n} .
$$

\subsection{Change of variables from $\left(x^{n}, y^{n}\right) \in \Omega^{n}$ to $\left(x^{0}, y^{0}\right) \in \Omega^{0}$}

As we proceed by a slow, small increment of the force, the virus is deformed and occupies a new configuration at each step. In order to avoid remeshing the domain, we choose here to make all the calculations in the initial domain $\Omega^{0}$. We need then to make a change of 
variables in the variational formulation (10). In the sequel we denote by $\tilde{\mathbf{u}}^{n}$ the displacement from $\Omega^{0}$ to $\Omega^{n}$. The variable will now be as follows:

$$
\begin{aligned}
& \varphi^{n}\left(\mathrm{x}^{0}\right)=\mathrm{x}^{n}, \quad \mathrm{x}^{0} \in \Omega^{0}, \mathrm{x}^{n} \in \Omega^{n} \\
& \varphi^{n+1}\left(\mathrm{x}^{0}\right)=\mathrm{x}^{n}+\mathrm{u}^{n}\left(\mathrm{x}^{n}\right)=\mathrm{x}^{0}+\tilde{\mathbf{u}}^{n}\left(\mathrm{x}^{0}\right)
\end{aligned}
$$

and we write:

$$
\begin{aligned}
& \mathbf{a}^{n}\left(\mathbf{u}^{n}, \mathbf{v}^{n}\right)=\mathbf{a}\left(\varphi^{n} ; \tilde{\mathbf{u}}^{n}, \tilde{\mathbf{v}}^{n}\right)=<\mathbf{A}\left(\varphi^{n}\right) \tilde{\mathbf{u}}^{n}, \tilde{\mathbf{v}}^{n}>_{\Omega^{0}}, \\
& \mathbf{l}^{n}\left(\mathbf{v}^{n}\right)=\mathbf{l}\left(\varphi^{n} ; \tilde{\mathbf{v}}^{n}\right)=<\mathbf{F}^{n+1}\left(\varphi^{n}\right), \tilde{\mathbf{v}}^{n}>_{\Gamma_{P}}+<\mathbf{f}_{a d h}\left(\varphi^{n}\right), \tilde{\mathbf{v}}^{n}>_{\Omega^{0}} .
\end{aligned}
$$

The problem (10) becomes

$$
\begin{aligned}
& \tilde{\mathbf{u}}^{n} \in K \text { and } \\
& \mathbf{a}\left(\varphi^{n} ; \tilde{\mathbf{u}}^{n}, \tilde{\mathbf{v}}^{n}\right)=\mathbf{l}\left(\varphi^{n} ; \tilde{\mathbf{v}}^{n}\right), \quad \forall \tilde{\mathbf{v}}^{n} \in K_{0},
\end{aligned}
$$

and we will now make explicit each term in (13).

\subsubsection{Computation of $\mathbf{a}^{n}\left(\mathbf{u}^{n}, \mathbf{v}^{n}\right)=\mathbf{a}\left(\varphi^{n} ; \tilde{\mathbf{u}}^{n}, \tilde{\mathbf{v}}^{n}\right)$}

We want to compute:

$$
\int_{\Omega^{n}} \varepsilon^{n}\left(\mathbf{u}^{n}\right): \varepsilon^{n}\left(\mathbf{v}^{n}\right) d \mathbf{x}^{n}=\int_{\Omega^{n}} 2 \mu \varepsilon_{i j}^{n}\left(\mathbf{u}^{n}\right) \varepsilon_{i j}^{n}\left(\mathbf{v}^{n}\right) d \mathbf{x}^{n}+\int_{\Omega^{n}} \lambda \varepsilon_{k k}^{n}\left(\mathbf{u}^{n}\right) \varepsilon_{i i}^{n}\left(\mathbf{v}^{n}\right) d \mathbf{x}^{n} .
$$

We have

$$
\begin{aligned}
\int_{\Omega^{n}} 2 \mu \varepsilon_{i j}^{n}\left(\mathbf{u}^{n}\right) \varepsilon_{i j}^{n}\left(\mathbf{v}^{n}\right) d \mathbf{x}^{n} & =\mu \int_{\Omega^{n}}\left(u_{1, y^{n}}^{n}+u_{2, x^{n}}^{n}\right)\left(v_{1, y^{n}}^{n}+v_{2, x^{n}}^{n}\right) d \mathbf{x}^{n} \\
& +\frac{\mu}{2} \int_{\Omega^{n}}\left(u_{1, x^{n}}^{n} v_{1, x^{n}}^{n}+u_{2, y^{n}}^{n} v_{2, y^{n}}^{n}\right) d \mathbf{x}^{n}, \\
\int_{\Omega^{n}} \lambda \varepsilon_{k k}^{n}\left(\mathbf{u}^{n}\right) \varepsilon_{i i}^{n}\left(\mathbf{v}^{n}\right) d \mathbf{x}^{n}= & \lambda \int_{\Omega^{n}}\left(u_{1, x^{n}}^{n}+u_{2, y^{n}}^{n}\right)\left(v_{1, x^{n}}^{n}+v_{2, y^{n}}^{n}\right) d \mathbf{x}^{n} .
\end{aligned}
$$

We compute each term

$$
\begin{aligned}
\mu \int_{\Omega^{n}}\left(u_{1, y^{n}}^{n}+u_{2, x^{n}}^{n}\right)\left(v_{1, y^{n}}^{n}+v_{2, x^{n}}^{n}\right) d \mathbf{x}^{n} & =\mu \int_{\Omega^{0}}\left(u_{1, y^{n}}^{n}+u_{2, x^{n}}^{n}\right)\left(v_{1, y^{n}}^{n}+v_{2, x^{n}}^{n}\right)\left|\nabla \varphi^{n}(\mathbf{x})\right| d \mathbf{x} \\
\frac{\mu}{2} \int_{\Omega^{n}}\left(u_{1, x^{n}}^{n} v_{1, x^{n}}^{n}+u_{2, y^{n}}^{n} v_{2, y^{n}}^{n}\right) d \mathbf{x}^{n} & =\frac{\mu}{2} \int_{\Omega^{0}}\left(u_{1, x^{n}}^{n} v_{1, x^{n}}^{n}+u_{2, y^{n}}^{n} v_{2, y^{n}}^{n}\right)\left|\nabla \varphi^{n}(\mathbf{x})\right| d \mathbf{x}, \\
\lambda \int_{\Omega^{n}}\left(u_{1, x^{n}}^{n}+u_{2, y^{n}}^{n}\right)\left(v_{1, x^{n}}^{n}+v_{2, y^{n}}^{n}\right) d \mathbf{x}^{n} & =\lambda \int_{\Omega^{0}}\left(u_{1, x^{n}}^{n}+u_{2, y^{n}}^{n}\right)\left(v_{1, x^{n}}^{n}+v_{2, y^{n}}^{n}\right)\left|\nabla \boldsymbol{\varphi}^{n}(\mathbf{x})\right| d \mathbf{x} .
\end{aligned}
$$

We compute each derivative as follows:

$$
\begin{aligned}
& u_{1, x^{n}}^{n}=\tilde{u}_{1, x} \frac{\partial x}{\partial x^{n}}+\tilde{u}_{1, y} \frac{\partial y}{\partial x^{n}}, \quad u_{1, y^{n}}^{n}=\tilde{u}_{1, y} \frac{\partial y}{\partial y^{n}}+\tilde{u}_{1, x} \frac{\partial x}{\partial y^{n}} \\
& u_{2, x^{n}}^{n}=\tilde{u}_{2, x} \frac{\partial x}{\partial x^{n}}+\tilde{u}_{2, y} \frac{\partial y}{\partial x^{n}}, \quad u_{2, y^{n}}^{n}=\tilde{u}_{2, y} \frac{\partial y}{\partial y^{n}}+\tilde{u}_{2, x} \frac{\partial x}{\partial y^{n}}
\end{aligned}
$$


Since $\frac{\partial\left(x^{n}, y^{n}\right)}{\partial(x, y)}=\nabla \varphi^{n}$ we need to compute $\left(\nabla \varphi^{n}\right)^{-1}$ :

$$
\left(\nabla \varphi^{n}\right)^{-1}=\frac{1}{\left|\nabla \varphi^{n}\right|}\left[\begin{array}{cc}
\varphi_{2, y}^{n} & -\varphi_{1, y}^{n} \\
-\varphi_{2, x}^{n} & \varphi_{1, x}^{n}
\end{array}\right]
$$

and therefore

$$
\begin{aligned}
& \frac{\partial x}{\partial x^{n}}=\frac{\varphi_{2, y}^{n}}{\left|\nabla \varphi^{n}\right|}, \frac{\partial x}{\partial y^{n}}=-\frac{\varphi_{1, y}^{n}}{\left|\nabla \varphi^{n}\right|} \\
& \frac{\partial y}{\partial x^{n}}=-\frac{\varphi_{2, x}^{n}}{\left|\nabla \varphi^{n}\right|}, \quad \frac{\partial y}{\partial y^{n}}=\frac{\varphi_{1, x}^{n}}{\left|\nabla \varphi^{n}\right|} .
\end{aligned}
$$

Then (14) becomes:

$$
\begin{gathered}
<\mathbf{A}^{n} \mathbf{u}^{n}, \mathbf{v}^{n}>_{\Omega^{n}}=\mathbf{a}^{n}\left(\mathbf{u}^{n}, \mathbf{v}^{n}\right)=\mathbf{a}\left(\varphi^{n} ; \mathbf{u}^{n}, \mathbf{v}^{n}\right)=<\mathbf{A}\left(\varphi^{n}\right) \tilde{\mathbf{u}}^{n}, \tilde{\mathbf{v}}^{n}>_{\Omega^{0}}= \\
\int_{\Omega^{0}} \frac{\mu}{\left|\nabla \varphi^{n}\right|}\left(-\tilde{u}_{1, x} \varphi_{1, y}^{n}+\tilde{u}_{1, y} \varphi_{1, x}^{n}+\tilde{u}_{2, x} \varphi_{2, y}^{n}-\tilde{u}_{2, y} \varphi_{2, x}^{n}\right) \\
+\int_{\Omega^{0}} \frac{\mu}{2\left|\nabla \varphi^{n}\right|}\left[\left(\tilde{u}_{1, x} \varphi_{2, y}^{n}-\tilde{u}_{1, y} \varphi_{2, x}^{n}\right)\left(\tilde{v}_{1, x} \varphi_{2, y}^{n}-\tilde{v}_{1, y} \varphi_{2, x}^{n}\right)\right. \\
\left.+\left(-\tilde{u}_{2, x} \varphi_{1, y}^{n}+\tilde{u}_{2, y} \varphi_{1, x}^{n}\right)\left(-\tilde{v}_{2, x} \varphi_{1, y}^{n}+\tilde{v}_{2, y} \varphi_{1, x}^{n}\right)\right] d \mathbf{x} \\
+\int_{\Omega^{0}} \frac{\lambda}{\left|\nabla \varphi^{n}\right|}\left(\tilde{u}_{1, x} \varphi_{2, y}^{n}-\tilde{u}_{1, y} \varphi_{2, x}^{n}-\tilde{u}_{2, x} \varphi_{1, y}^{n}+\tilde{u}_{2, y} \varphi_{1, x}^{n}\right) \\
\left(\tilde{v}_{1, x} \varphi_{2, y}^{n}-\tilde{v}_{1, y} \varphi_{2, x}^{n}-\tilde{v}_{2, x} \varphi_{1, y}^{n}+\tilde{v}_{2, y} \varphi_{1, x}^{n}\right) d \mathbf{x} .
\end{gathered}
$$

\subsubsection{Computation of the virtual power of the punch force}

The virtual power of the punch force for a virtual displacement $\mathbf{v}^{n}$ is:

$$
<\mathbf{F}^{n+1}, \mathbf{v}^{n}>_{\Gamma_{P}^{n}}=<\mathbf{F}^{n+1}\left(\varphi^{n}\right), \tilde{\mathbf{v}}^{n}>_{\Gamma_{P}} .
$$

In our case $\mathbf{F}^{n+1}=-\frac{n+1}{N} F \mathbf{n}$, where $F=$ constant and $\mathbf{n}$ is the outward normal vector to $\Gamma_{p}^{n}$. Therefore, the equation yields

$$
<\mathbf{F}^{n+1}, \mathbf{v}^{n}>_{\Gamma_{P}^{n}}=-\frac{n+1}{N} \int_{\Gamma_{P}^{n}} F \mathbf{n} \cdot \mathbf{v}^{n} d \mathbf{x}^{n}=-\frac{n+1}{N} F \int_{\Gamma_{P}}\left(\mathbf{n} \circ \varphi^{n}\right) \cdot \tilde{\mathbf{v}}^{n}\left|\nabla \varphi^{n}\right| d \mathbf{x} .
$$

Thus we define

$$
\mathbf{F}^{n+1}\left(\boldsymbol{\varphi}^{n}\right)=-\frac{(n+1) F}{N}\left(\mathbf{n} \circ \varphi^{n}\right)\left|\nabla \varphi^{n}\right|
$$




\subsubsection{Computation of the virtual power of the adhesion forces}

We give now the definition of the adhesion force resulting from the interaction between the molecules of the virus and the molecules of the contact surface. In this study we choose the adhesion force derived from the Lennard-Jones potential defined (see [11]) by:

$$
U_{a d h}^{n}:=\int_{-\infty}^{-r_{2}-r_{0}} \int_{-\infty}^{+\infty} w(\mathbf{r}) d x^{l} d y^{l},
$$

where,

$$
\begin{aligned}
& \mathbf{r}=\left(x^{l}-x^{\varphi}, y^{l}-x^{\varphi}\right) \\
& r_{0}=0.5 \mathrm{~nm} .
\end{aligned}
$$

The definition of $\mathbf{r}$ comes from the fact that we want $\|\mathbf{r}\| \geq r_{0}=0.5 \mathrm{~nm}$, because the virus can not rest directly on the surface and it stays at the minimal distance $r_{0}$ from the surface. We set the origin at the center of the virus and define the support at $y=-r_{2}-r_{0}$. Figure 1 shows the different coordinates.

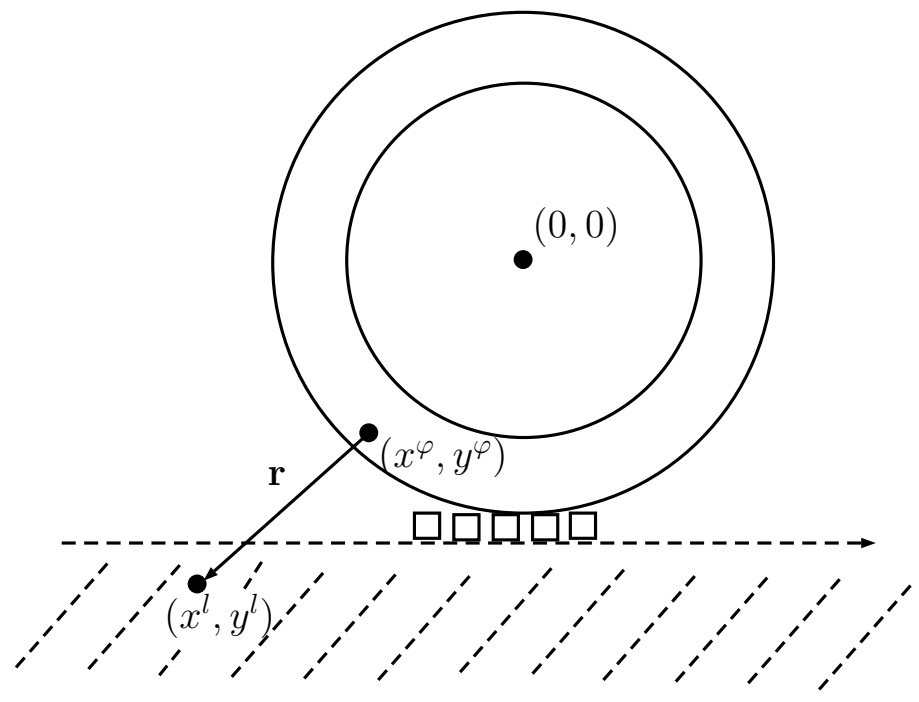

Figure 1: Adhesion forces.

The function $w$ is given by

$$
w(\mathbf{r})=\frac{A}{|\mathbf{r}|^{12}}-\frac{B}{|\mathbf{r}|^{6}} .
$$

Remark 2 To find $A$ and $B$ we have to solve

$$
\begin{aligned}
& |\mathbf{r}|=0.5 \Longrightarrow \frac{d w}{d|\mathbf{r}|}=0 \text { and } w=-5.0 \cdot k T, \\
& |\mathbf{r}|<0.25 \Longrightarrow w \gg k T,
\end{aligned}
$$


where $k$ is the Boltzmann constant and $T$ is the temperature.

Therefore with $k=1.3806488 \cdot 10^{-23} \mathrm{~m}^{2} \mathrm{~kg} \mathrm{~s}^{-2} \mathrm{~K}^{-1}=1.3806488 \cdot 10^{-5} \mathrm{~nm}^{2} \mathrm{~kg} \mathrm{~s}^{-2} \mathrm{~K}^{-1}$, and $T=310.1 K(=37 \quad C)$ we want to solve

$$
\left\{\begin{array}{l}
-\frac{12 A}{r_{0}^{12}}+\frac{6 B}{r_{0}^{6}}=0, \\
\frac{A}{r_{0}^{12}}-\frac{B}{r_{0}^{6}}=-5 k T .
\end{array}\right.
$$

We obtain:

$$
A=5 r_{0}^{12} k T, \quad B=10 r_{0}^{6} k T
$$

and $w$ becomes

$$
w(\mathbf{r})=\frac{5 r_{0}^{12} k T}{|\mathbf{r}|^{12}}-\frac{10 r_{0}^{6} k T}{|\mathbf{r}|^{6}}
$$

Now to find the adhesion force we write the potential as

$$
U_{a d h}^{n}=\int_{-\infty}^{\infty}\left[\frac{A}{\left.\left(\left(x^{l}-x^{n}\right)^{2}+\left(y^{l}-y^{n}\right)\right)^{2}\right)^{6}}-\frac{B}{\left.\left(\left(x^{l}-x^{n}\right)^{2}+\left(y^{l}-y^{n}\right)\right)^{2}\right)^{3}}\right] d x_{l} .
$$

So the adhesion force is

$$
\mathbf{f}_{a d h}^{n}:=-\nabla U_{a d h}^{n}=-\left(\frac{\partial U_{a d h}^{n}}{\partial x^{n}}, \frac{\partial U_{a d h}^{n}}{\partial y^{n}}\right)^{t},
$$

which reads

$$
\mathbf{f}_{a d h}^{n}:=\left(\begin{array}{l}
\int_{-r_{2}-r_{0}}^{-\infty} \int_{-\infty}^{\infty}\left[-\frac{12 A\left(x^{l}-x^{n}\right)}{\left.\left(\left(x^{l}-x^{n}\right)^{2}+\left(y^{l}-y^{n}\right)\right)^{2}\right)^{7}}+\frac{6 B\left(x^{l}-x^{n}\right)}{\left.\left(\left(x^{l}-x^{n}\right)^{2}+\left(y^{l}-y^{n}\right)\right)^{2}\right)^{4}}\right] d x^{l} d y^{l} \\
\int_{-r_{2}-r_{0}}^{-\infty} \int_{-\infty}^{\infty}\left[-\frac{\left.12 A\left(y^{l}-y^{n}\right)\right)}{\left.\left(\left(x^{l}-x^{n}\right)^{2}+\left(y^{l}-y^{n}\right)\right)^{2}\right)^{7}}+\frac{\left.6 B\left(y^{l}-y^{n}\right)\right)}{\left.\left(\left(x^{l}-x^{n}\right)^{2}+\left(y^{l}-y^{n}\right)\right)^{2}\right)^{4}}\right] d x^{l} d y^{l}
\end{array}\right) .
$$

So we have:

$$
<\mathbf{f}_{a d h}^{n}, \mathbf{v}^{n}>_{\Omega^{n}}=\int_{\Omega^{n}} \mathbf{f}_{a d h}^{n} \cdot \mathbf{v}^{n} d \mathbf{x}^{n} .
$$

By changing the variables we obtain:

$$
<\mathbf{f}_{a d h}^{n}\left(\varphi^{n}\right), \tilde{\mathbf{v}}^{\mathbf{n}}>_{\Omega^{0}}=\int_{\Omega^{0}}\left(\mathbf{f}_{a d h}^{n} \circ \varphi^{n}\right) \cdot \tilde{\mathbf{v}}^{\mathbf{n}}\left|\nabla \varphi^{n}\right| d \mathbf{x}
$$

where $\tilde{\mathbf{v}}^{\mathbf{n}}$ is a virtual displacement from $\Omega^{0}$ to $\Omega^{n}$. 


\section{$3 \quad$ Numerical approximation}

Let us recall the equation we are solving at each step $n$, for $0 \leq n<N$ :

$$
\begin{array}{r}
<\mathbf{A}\left(\varphi^{n}\right) \tilde{\mathbf{u}}^{n}, \tilde{\mathbf{v}}>_{\Omega^{0}}=<\mathbf{F}^{n+1}\left(\varphi^{n}\right), \tilde{\mathbf{v}}>_{\Gamma_{P}}+<\mathbf{f}_{a d h}\left(\varphi^{n}\right), \tilde{\mathbf{v}}>_{\Omega^{0}}, \\
\forall \tilde{\mathbf{v}} \in K_{0} .
\end{array}
$$

In the following section we will present the discrete version of this equation.

\subsection{Finite elements}

In this section we will drop the index $n$ to construct the finite element basis and we will write the discrete version of the operator $\mathbf{A}$. Let $\mathcal{T}_{h}$ be a triangulation of $\Omega^{0}$, containing $N_{T}$ triangles and $N_{v}$ vertices, as in the example shown in Figure 2. Let

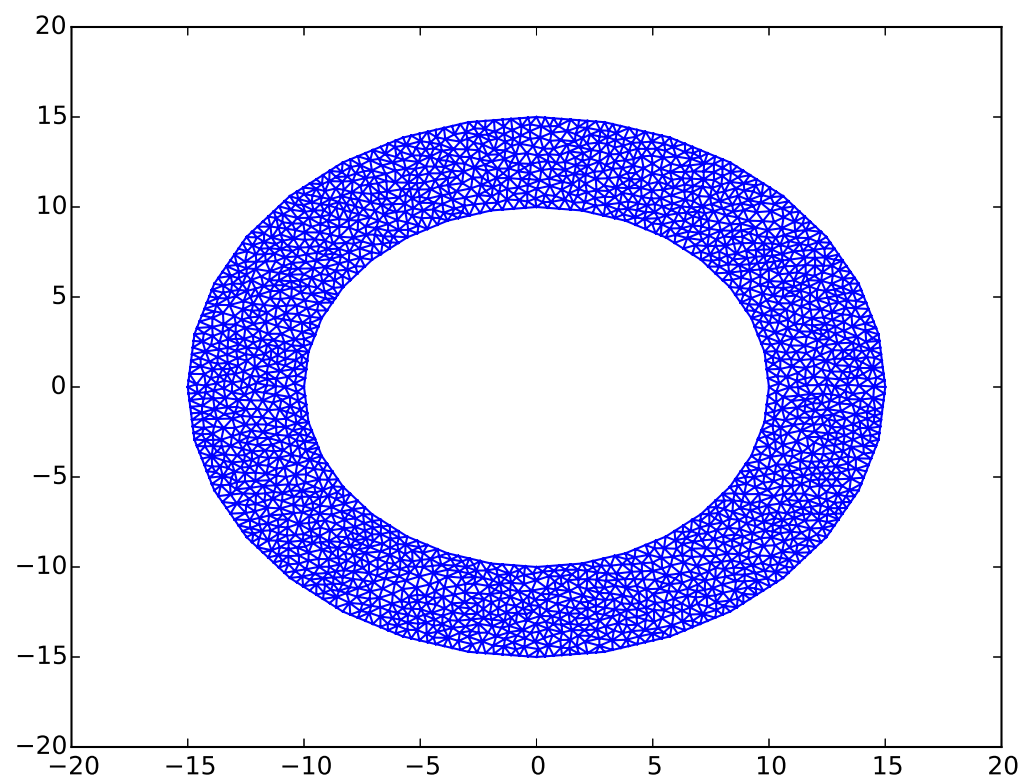

Figure 2: Example of triangulation of $\Omega$

$$
\mathcal{V}_{h}=\left\{\boldsymbol{\psi}_{h} \in H^{1}(\Omega)\left|\boldsymbol{\psi}_{h}\right|_{\tau} \in \mathbb{P}_{1} \forall \tau \in \mathcal{T}_{h}\right\}
$$

then we define the basis of $\mathcal{V}_{h}\left\{\boldsymbol{\psi}_{i}\right\}_{1 \leq i \leq N_{v}}$. For $\mathbf{u}_{h} \in \mathcal{V}_{h}$, we can write the discretized version of $\mathbf{a}(\varphi)$ and $\mathbf{l}(\varphi)$ on $\Omega^{0}$, that is for $\varphi_{h} \in \mathcal{V}_{h}$ and $\mathbf{u}_{h} \in \mathcal{V}_{h}$

$$
\left(\mathbf{A}\left(\varphi_{h}\right) \mathbf{u}_{h}, \mathbf{u}_{h}\right)=\mathbf{a}\left(\varphi_{h} ; \mathbf{u}_{h}, \mathbf{u}_{h}\right)
$$

therefore $\mathbf{A}_{i j}\left(\boldsymbol{\varphi}_{h}\right)=\mathbf{a}\left(\boldsymbol{\varphi}_{h} ; \boldsymbol{\psi}_{i}, \boldsymbol{\psi}_{j}\right)$, and we also define

$$
\mathbf{b}\left(\varphi_{h}\right)=\mathbf{l}\left(\varphi_{h} ; \mathbf{u}_{h}\right) .
$$


Remark 3 The construction of the basis is done as follows. Let $\left\{T_{i}\right\}_{1 \leq i \leq B_{T}}$ be the triangles of $\Omega^{0}$ and we suppose that we have $N_{v}$ vertices. We write $\mathbf{x}_{i}=\left(x_{i}, y_{i}\right)$. Then we have:

$$
\begin{aligned}
& \psi_{i}^{1}=\left(0, \boldsymbol{\psi}_{i}\right), \text { for } 1 \leq i \leq N_{v}, \\
& \psi_{i}^{2}=\left(\boldsymbol{\psi}_{i}, 0\right), \text { for } 1 \leq i \leq N_{v} .
\end{aligned}
$$

Therefore

$$
\mathbf{u}=\left(u^{1}, u^{2}\right):=\sum_{i=1}^{N_{v}} u_{i}^{1} \boldsymbol{\psi}_{i}^{1}+\sum_{j=1}^{N_{v}} u_{j}^{2} \boldsymbol{\psi}_{j}^{2} .
$$

Now to define $\boldsymbol{\psi}$ on a triangle $T_{k}$, we define the three vertices of this triangle by the indices 1, 2, 3 and from [13] we have

$$
\begin{aligned}
& H_{1}=\frac{1}{2\left|T_{k}\right|}\left[\left(x_{2} y_{3}-x_{3} y_{2}\right)+\left(y_{2}-y_{3}\right) x+\left(x_{3}-x_{2}\right) y\right], \\
& H_{2}=\frac{1}{2\left|T_{k}\right|}\left[\left(x_{3} y_{1}-x_{1} y_{3}\right)+\left(y_{3}-y_{1}\right) x+\left(x_{1}-x_{3}\right) y\right], \\
& H_{3}=\frac{1}{2\left|T_{k}\right|}\left[\left(x_{1} y_{2}-x_{2} y_{1}\right)+\left(y_{1}-y_{2}\right) x+\left(x_{2}-x_{1}\right) y\right],
\end{aligned}
$$

where

$$
\left|T_{k}\right|=\frac{1}{2} \operatorname{det}\left[\begin{array}{lll}
1 & x_{1} & y_{1} \\
1 & x_{2} & y_{2} \\
1 & x_{3} & y_{3}
\end{array}\right] .
$$

With these definitions we have

$$
\left.u^{1}\right|_{T_{k}}=u_{1}^{1} H_{1}(x, y)+u_{2}^{1} H_{2}(x, y)+u_{3}^{1} H_{3}(x, y) ;
$$

same for $u_{2}^{1}, u_{3}^{1}, u_{1}^{2}, u_{2}^{2}$, and $u_{3}^{2}$.

For our numerical simulations we will use two different stands: one flat stand, see Figure 3 , and a stand made of two circles of $60 \mathrm{~nm}$ radius, see Figure 4 . 


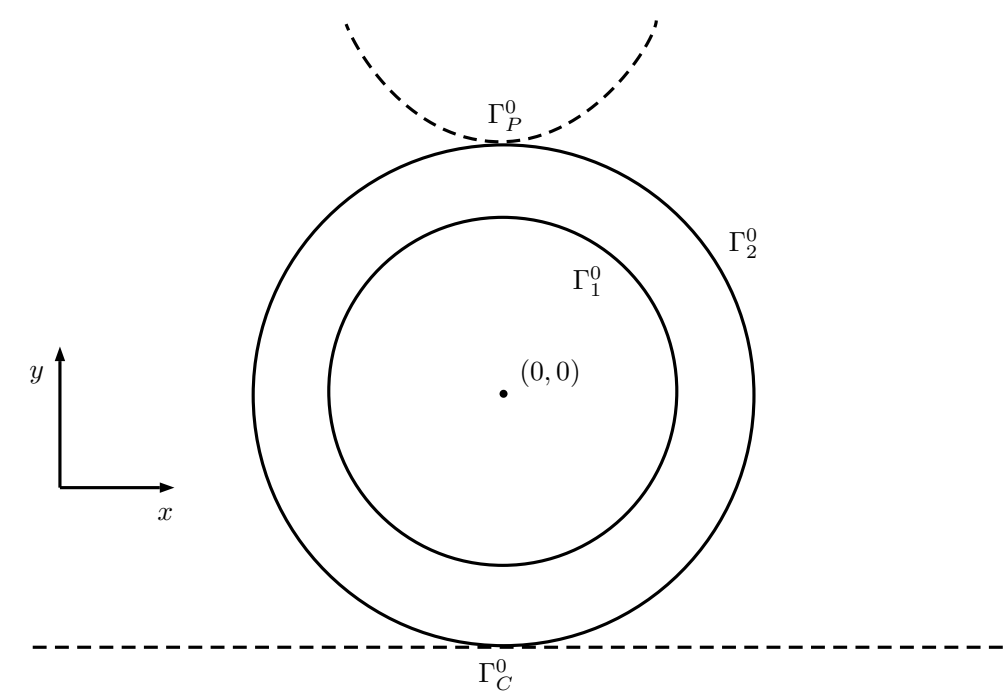

Figure 3: Domain $\Omega^{0}$ with puncher and flat stand.

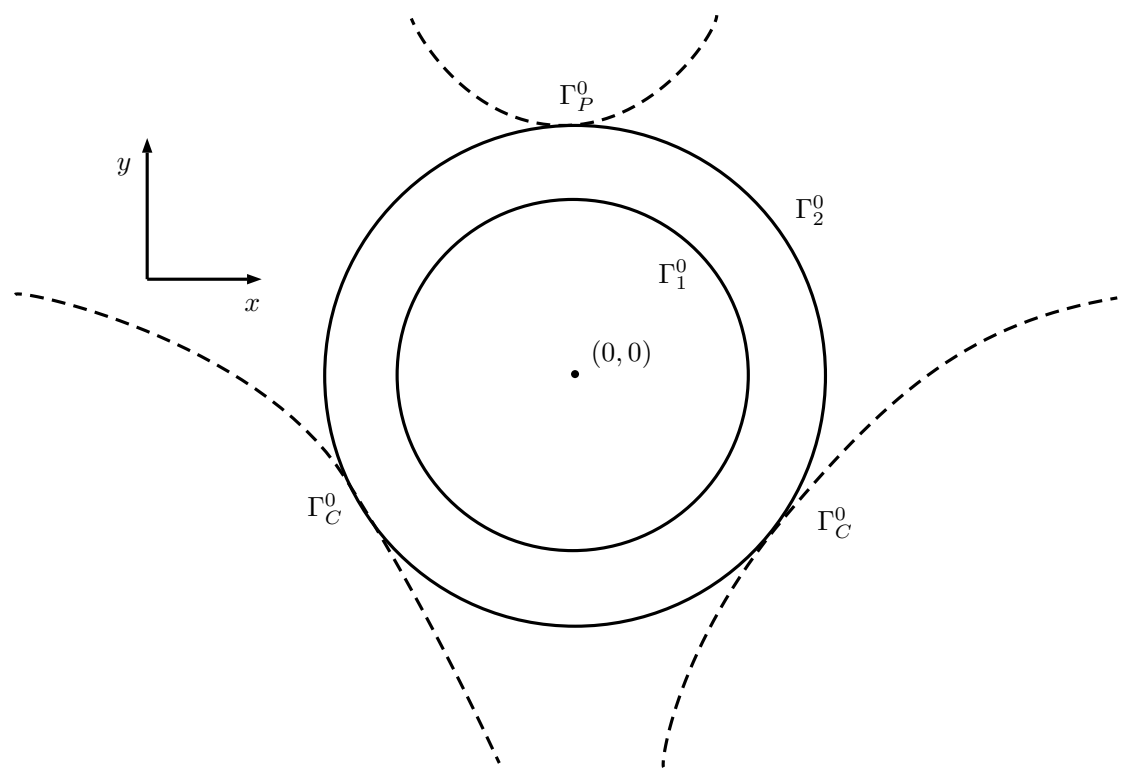

Figure 4: Domain $\Omega^{0}$ with puncher and circle stands.

On the flat stand we have to impose the displacement and the rotation at one point in order to have a unique solution; on the two circles stand we fix the displacement at two points which will also fix the rotation. In the following we explain which points we choose.

In the case of the flat support, we fix the vertex that is in contact with the support at iteration 0 and we fix the horizontal translation of the point in contact with the puncher at iteration 0 . Let us assume that $\left(x_{i}, y_{i}\right)$, for $1 \leq i \leq N_{v}$ are the vertices of our mesh on $\Omega^{0}$. 
We define

$$
i_{\text {top }}=\operatorname{argmax}\left(y_{i}\right), \quad i_{\text {bottom }}=\operatorname{argmin}\left(y_{i}\right) .
$$

We then fix the point at $i_{\text {bottom }}$ and fix the horizontal translation in $x$ at $i_{\text {top }}$.

In the case of circles support, we fix the two vertices in contact with each circle at iteration 0 . Let $\left(x_{l}, y_{l}\right)=\left(-\frac{75}{\sqrt{2}},-\frac{75}{\sqrt{2}}\right)$ be the center of the left circle and let $\left(x_{r}, y_{r}\right)=\left(\frac{75}{\sqrt{2}},-\frac{75}{\sqrt{2}}\right)$ be the center of the right circle. We then fix the displacement at

$$
i_{\text {left }}=\operatorname{argmin}\left(\sqrt{\left(x_{i}-x_{l}\right)^{2}+\left(y_{i}-y_{l}\right)^{2}}\right), \quad i_{\text {right }}=\operatorname{argmin}\left(\sqrt{\left(x_{i}-x_{r}\right)^{2}+\left(y_{i}-y_{r}\right)^{2}}\right) .
$$

\subsection{Algorithm}

As explained before in order to solve the equations with a linear model we are proceeding by increments of the constant $F$ (and therefore the vector $\mathbf{F}$ ). Let $N$ be the total number of increments; we recall that the force exerted by the puncher on $\Gamma_{P}^{n}$ is

$$
\mathbf{F}^{n+1}=-\frac{n+1}{N} F \mathbf{n}, \text { for } 1 \leq n \leq N,
$$

where $\mathbf{n}$ is the outer normal vector and $\Omega^{n}$ is the domain at iteration $n, \varphi_{h}^{n}$ is the deformation from $\Omega^{0}$ to $\Omega^{n}$, and $\tilde{\mathbf{u}}_{h}^{n}$ is the displacement from $\Omega^{0}$ to $\Omega^{n}$. Recall that we have $\varphi_{h}^{n+1}=\mathbf{x}_{\mathbf{0}}+\tilde{\mathbf{u}}_{h}^{n}$, where $\mathbf{x}_{0}$ is the the identity function on $\Omega_{0}$. With these notation in mind we have at each step $n=0, \ldots, N-1$ :

$$
\begin{array}{r}
<\mathbf{A}\left(\varphi^{n}\right) \tilde{\mathbf{u}}_{h}^{n}, \tilde{\mathbf{v}}_{\mathbf{h}}>_{\Omega^{0}}=<\mathbf{F}^{n+1}\left(\varphi_{h}^{n}\right), \tilde{\mathbf{v}}_{\mathbf{h}}>_{\Gamma_{P}}+<\mathbf{f}_{a d h}\left(\varphi_{h}^{n}\right), \tilde{\mathbf{v}}_{\mathbf{h}}>_{\Omega^{0}}, \\
\forall \tilde{\mathbf{v}}_{\mathbf{h}} \in \mathcal{V}_{h} .
\end{array}
$$

For the sake of notational simplicity, we set

$$
\left\{\begin{array}{lr}
\mathcal{F}^{n+1}\left(\varphi^{n}\right)=F^{n+1}\left(\varphi_{h}^{n}\right) & \text { on } \Gamma_{P} \\
\mathcal{F}^{n+1}\left(\varphi^{n}\right)=0 & \text { on } \Omega^{0} \backslash \Gamma_{P}
\end{array}\right.
$$

Now our algorithm proceeds as follows: $\varphi_{h}^{0}=\mathbf{I d}$ is known. Then for $n=0, \ldots, N-1$, we assume that $\varphi_{h}^{n}$ is known and compute $\tilde{\mathbf{u}}_{h}^{n}$ :

1. Fix $\varphi^{n}$ and compute $\tilde{\mathbf{u}}^{n}$ using a Restart GMRES method on

$$
\mathbf{A}\left(\varphi_{h}^{n}\right) \mathbf{u}_{h}^{n+1}=\mathcal{F}^{n}\left(\varphi^{n}\right)+\mathbf{f}_{a d h}\left(\varphi_{h}^{n}\right) .
$$

2. Then we compute $\varphi^{n+1}=\mathbf{x}_{0}+\tilde{\mathbf{u}}^{n}$.

We are then ready to compute the next step starting from $\varphi^{n+1}$. 


\section{$4 \quad$ Numerical simulations}

In this section, we present the results of 3 simulations. Simulations 1 and 2 are done on the flat stands, but for Simulation 1 the adhesion forces are 0 so that the virus deforms only because of $\mathbf{F}$ and for Simulation 2 the adhesion forces are present. Simulation 3 is done on the two circles without any adhesion forces. Adhesion was not included at this point to allow the separate analysis of the influence of substrate geometry.

\subsection{Simulation 1: on the flat support without adhesion forces}

Our first simulation, on a flat support, is done with $F=0.022 \mathrm{nN}, \mathbf{f}_{a d h}=0$ and $N=30$. Figure 5 shows the results of the shape of the virus for the values of $F=00514,0.01027,0.0154,0.02054$ $\mathrm{nN}$.

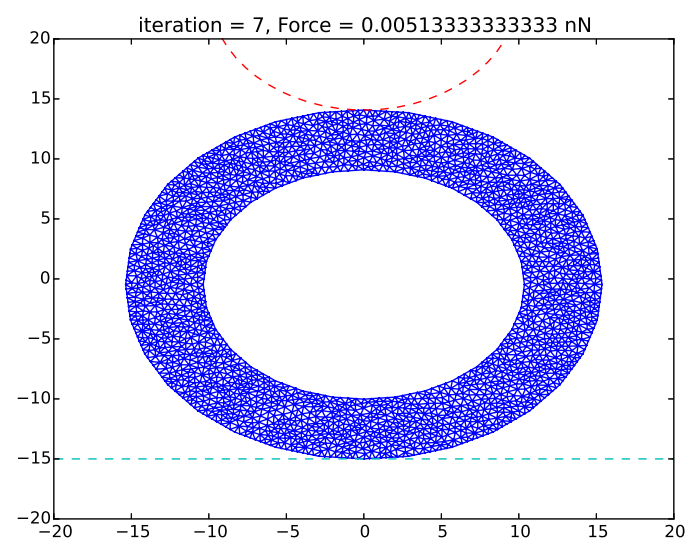

(a)

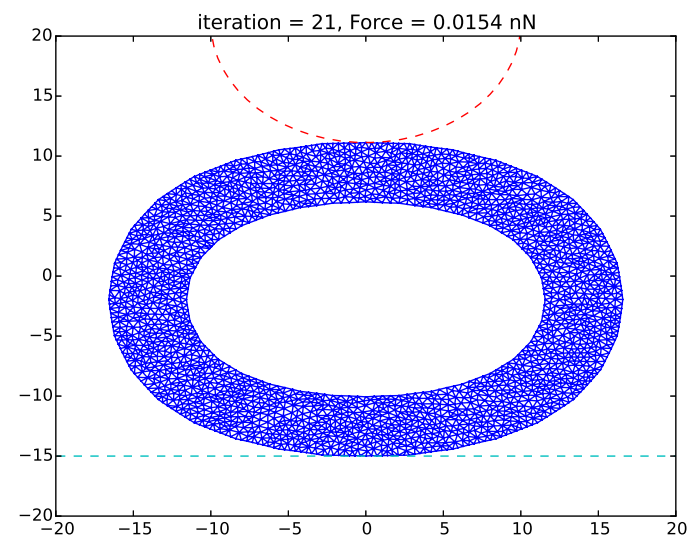

(c)

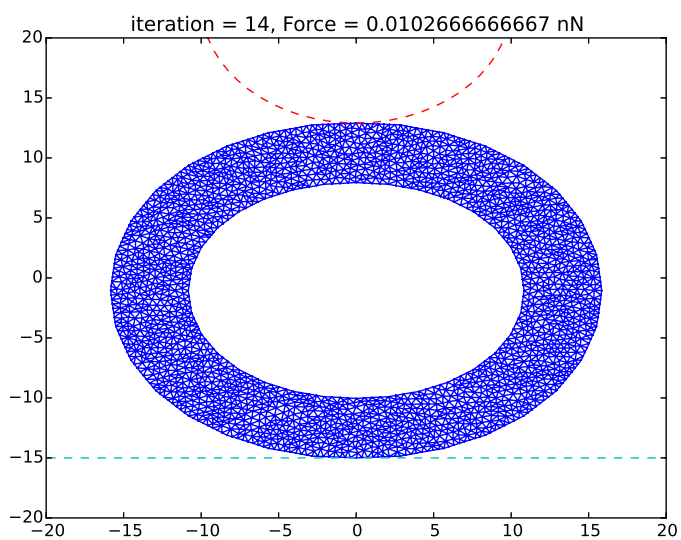

(b)

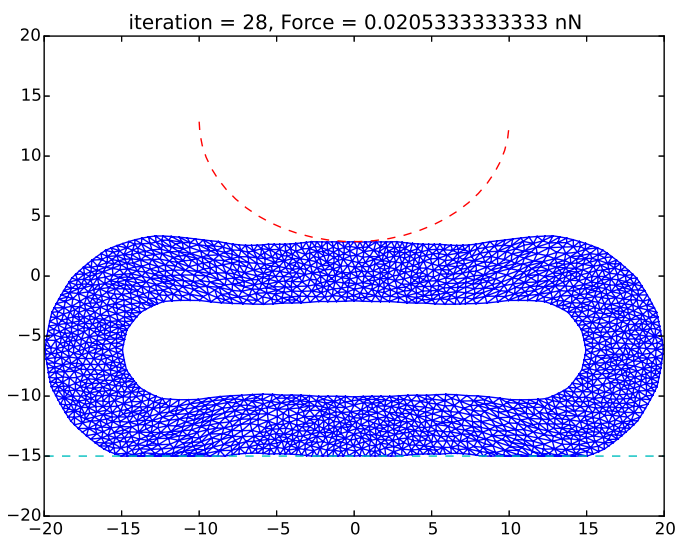

(d)

Figure 5: Shape of the shell at iteration 0, 15, 30, and 38 on a flat support. This corresponds to $F=00514,0.01027,0.0154,0.02054 \mathrm{nN}$. 


\subsection{Simulation 2: on the flat support with adhesion forces}

The following simulation is done on a flat support with $F=0.006, \mathbf{f}_{a d h}$ is defined as in (34). Figure 6 shows the results of the shape of the virus shell for the value of $F=$ $00514,0.01027,0.0154,0.02054 \mathrm{nN}$.

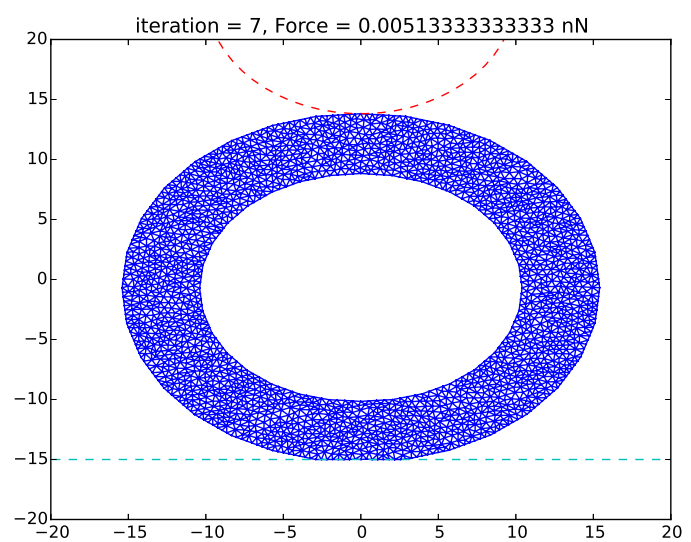

(a)

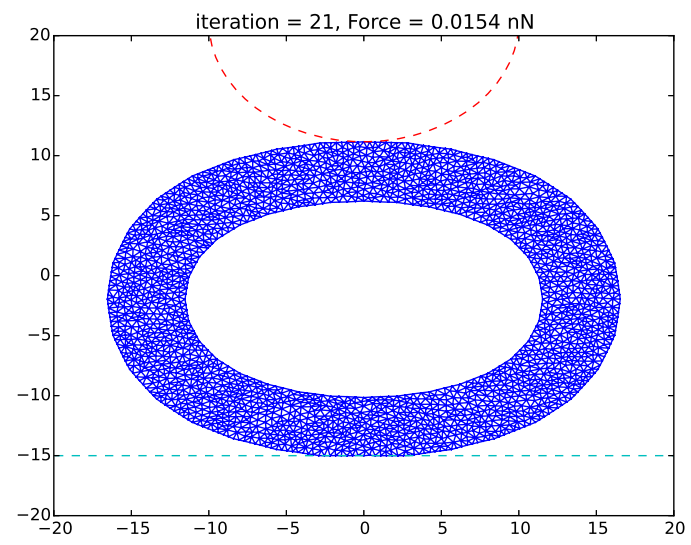

(c)

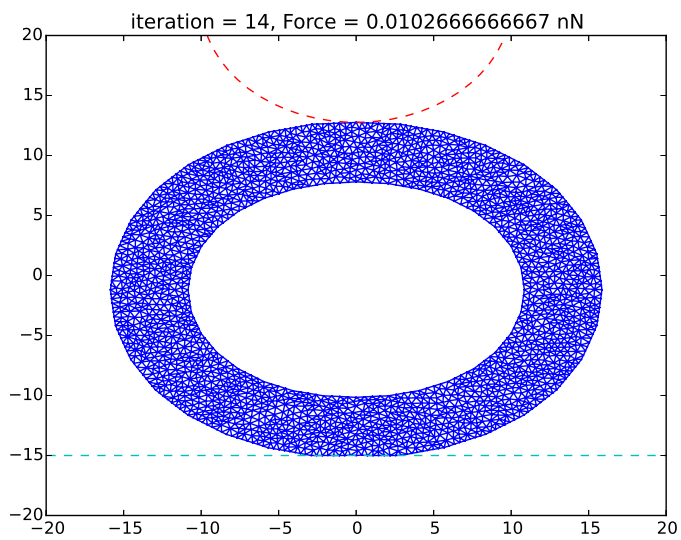

(b)

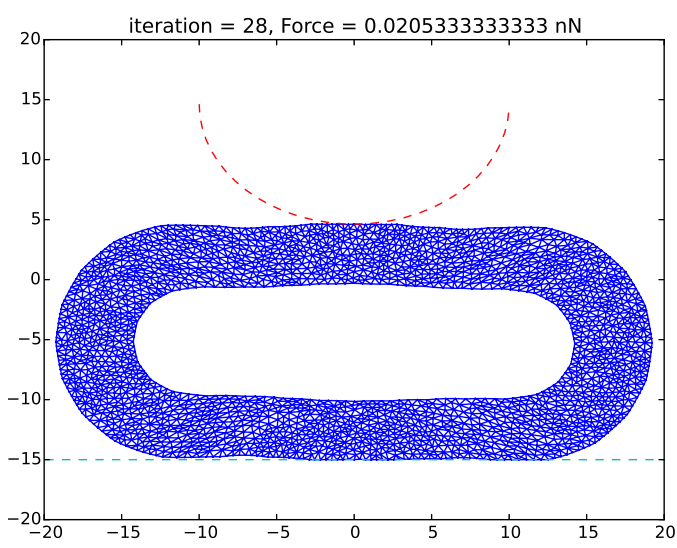

(d)

Figure 6: Shape of the shell at iteration $0,2,4$, and 6 on a flat support with adhesion forces. This corresponds to $F=00514,0.01027,0.0154,0.02054 \mathrm{nN}$. 


\subsection{Simulation 3: on the two circles support without adhesion forces}

This simulation is done on two circles as support with $F=0.03 \mathrm{nN}, \mathbf{f}_{a d h}=0$, and $N=30$. Figure 7 shows the results of the shape of the virus that correspond to the values for $F=$ $0.06,0.012,0.018,0.024 \mathrm{nN}$.

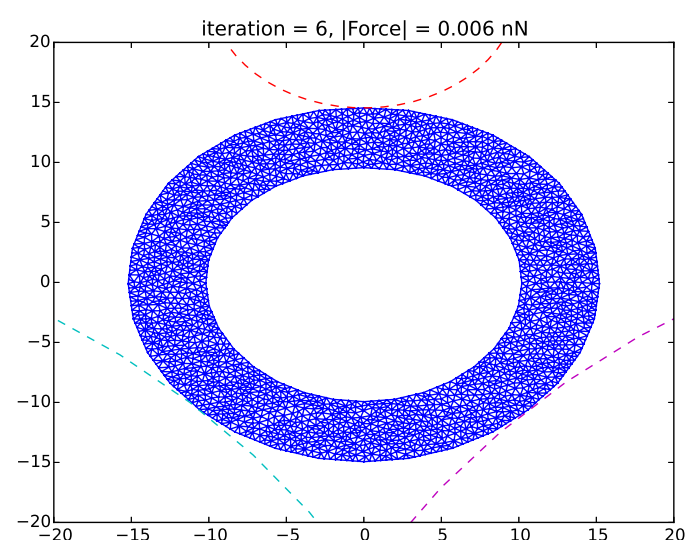

(a)

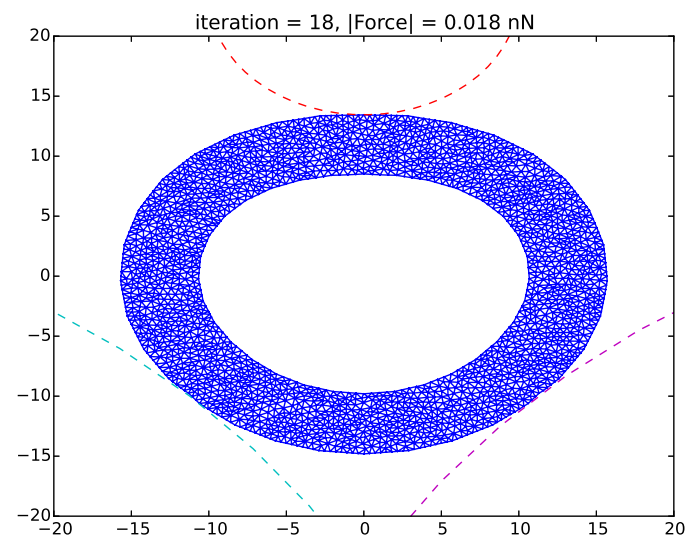

(c)

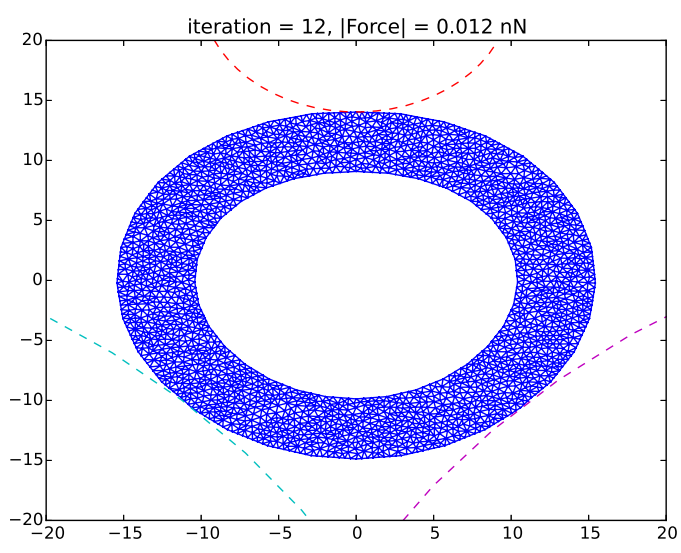

(b)

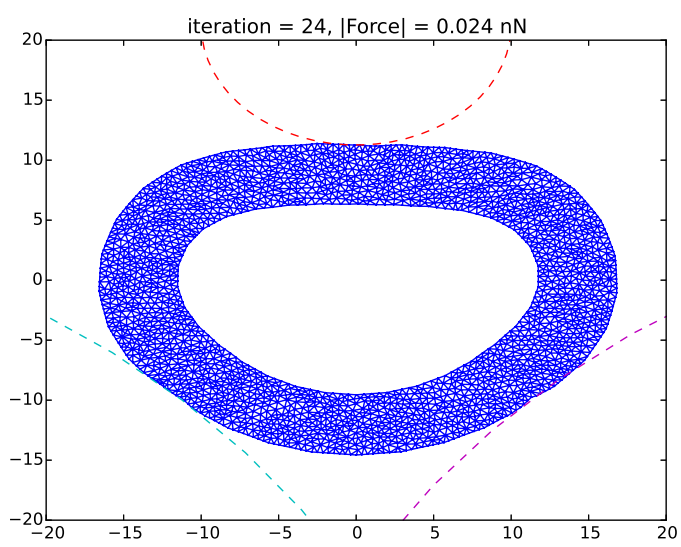

(d)

Figure 7: Shape the virus at iteration 0, 15, 30, and 36 on circles support. This corresponds to $F=0.06,0.012,0.018,0.024 \mathrm{nN}$.

Remark 4 Alternatively we may see the transformation $\Omega^{0} \longrightarrow \Omega=\Omega^{N}$ as a whole, where the unknowns are both the final state (given by $\varphi=\varphi^{N}, \varphi\left(\Omega^{0}\right)=\Omega$ with displacements $\left.\left.\mathbf{u}\left(\mathbf{x}^{0}\right)\right)=\varphi\left(\mathbf{x}^{0}\right)-\mathbf{x}^{0}\right)$, and the distribution of stresses $\boldsymbol{\sigma}$ in $\Omega$ (expressed in either $\Omega^{0}$ or $\Omega$ ). Here the mechanical equations are meant to be written in the deformed space $\Omega=\Omega^{N}$ which is unknown. We leave this problem for future studies as it pertains to nonlinear elasticity with large deformation [4], or possibly to some other model of nonlinear mechanics. 
For example, we can also deal directly with a nonlinear model for which

$$
\varepsilon_{i, j}=\frac{1}{2}\left(u_{i, j}+u_{j, i}+u_{k, i} u_{k, j}\right),
$$

and in our algorithm we would consider

$$
\varepsilon_{i, j}=\frac{1}{2}\left(u_{i, j}^{n}+u_{j, i}^{n}+u_{k, i}^{n} u_{k, j}^{n-1}\right) .
$$

Figure 8 shows how much the virus has been displaced with respect to the force $\mathbf{F}$ for the three simulations considered. For reasonable adhesion magnitude and the flat stand, the displacement - force curves are very similar overall between the case with and without adhesion. Only the initial deformation and the area of contact with the flat support are somewhat different because of the adhesion forces. However, the force-displacement curve from simulation of indentation on a substrate modeled by two adjacent circles is significantly different from that of the flat stand.

\section{Conclusion}

In conclusion, we have performed finite-element simulations of the deformation of an elastic shell on a substrate with emphasis on the role on the substrate-shell interaction. For the first time, adhesion interactions between the substrate and the shell being indented by a normal distributed force were considered in a model applicable to recent experiments of AFM indentation of virus particles. For typical nanoparticle-substrate adhesion forces, with the exception of small differences at initial indentations, the force-displacement curves are similar with and without adhesion. Therefore, adhesion on a flat surface is not expected to affect measurements of the elastic constant for viruses in any appreciable way. However, when the substrate is not flat, like in the case of the virus shell being lodged in a circular cusp, the apparent stiffness measured by indentation is expected to be significantly different.

\section{Acknowledgment}

This research was supported in part by the National Science Foundation under the grants NSF-DMS-1206438, NSF-DMS-1510249 and by the Research Fund of Indiana University. 


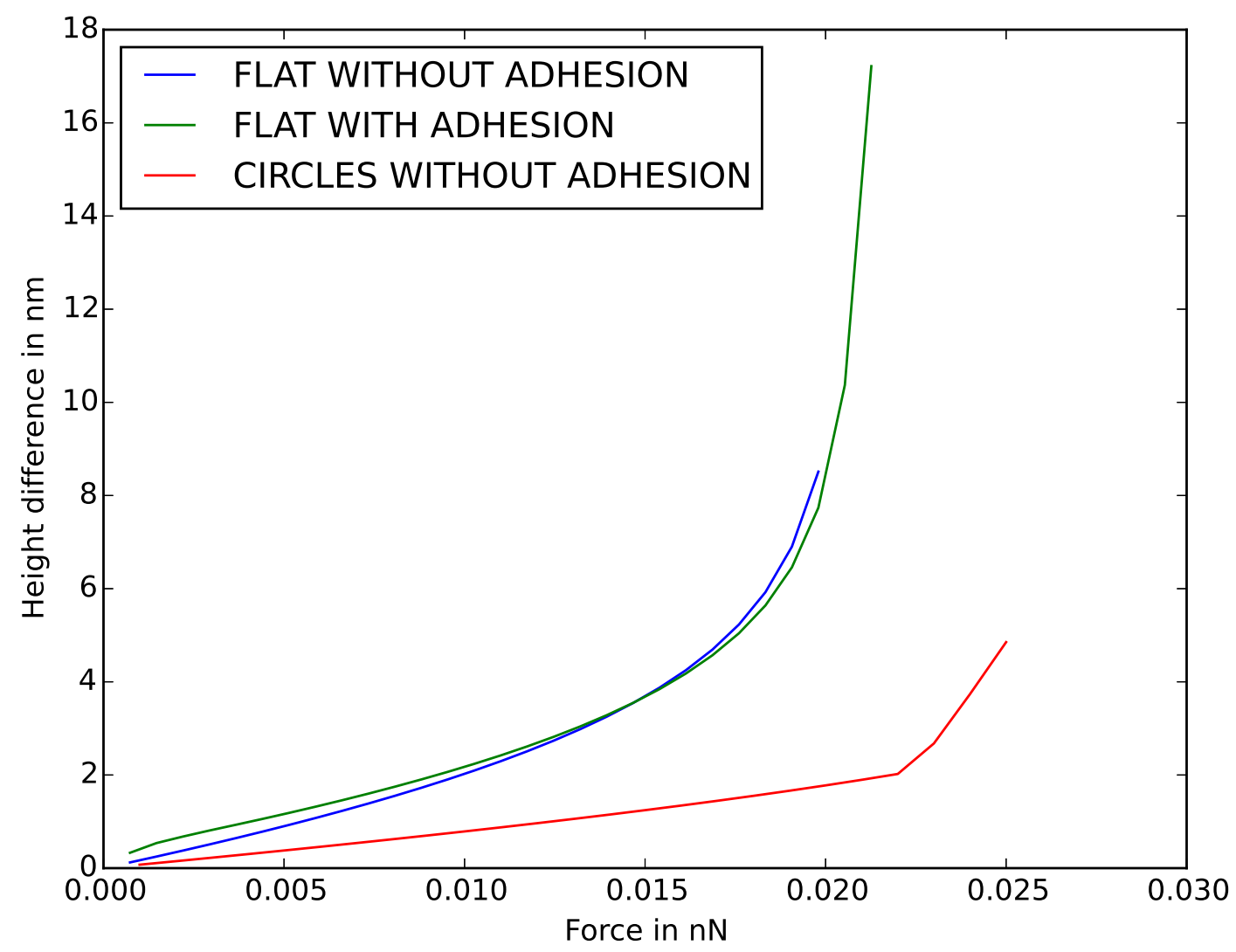

Figure 8: The height displacement of the virus in our three simulations.

\section{References}

[1] Aylin Ahadi, Dan Johansson, and Alex Evilevitch.

Modeling and simulation of the mechanical response from nanoindentation test of DNAfilled viral capsids.

Journal of biological physics, 39(2):183-99, mar 2013.

[2] C Bustamante, J C Macosko, and G J L Wuite.

Grabbing the cat by the tail: Manipulating molecules one by one.

Nature Reviews Molecular Cell Biology, 1(2):130-136, nov 2000.

[3] C. Carrasco, A. Carreira, I. A. T. Schaap, P. A. Serena, J. Gmez-Herrero, M. G. Mateu, and P. J. de Pablo.

Dna-mediated anisotropic mechanical reinforcement of a virus.

Proceedings of the National Academy of Sciences, 103(37):13706-13711, 2006.

[4] Philippe G. Ciarlet. 
Mathematical elasticity. Vol. I, volume 20 of Studies in Mathematics and its Applications.

North-Holland Publishing Co., Amsterdam, 1988.

Three-dimensional elasticity.

[5] Marek Cieplak and Mark O Robbins.

Nanoindentation of 35 virus capsids in a molecular model: relating mechanical properties to structure.

PloS one, 8(6):e63640, jan 2013.

[6] G. Duvaut and J.-L. Lions.

Inequalities in mechanics and physics.

Springer-Verlag, Berlin-New York, 1976.

Translated from the French by C. W. John, Grundlehren der Mathematischen Wissenschaften, 219.

[7] Gaetano Fichera.

Problemi elastostatici con vincoli unilaterali: Il problema di Signorini con ambigue condizioni al contorno.

Atti Accad. Naz. Lincei Mem. Cl. Sci. Fis. Mat. Natur. Sez. I(8), 7:91-140, 1963/1964.

[8] L. H. Ford.

Estimate of the vibrational frequencies of spherical virus particles.

Phys. Rev. E. Stat. Nonlin. Soft. Matter. Phys., 67:1539-3755, May 2003.

[9] Melissa M. Gibbons and William S. Klug.

Nonlinear finite-element analysis of nanoindentation of viral capsids.

Phys. Rev. E, 75:031901, Mar 2007.

[10] Mercedes Hernando-Prez, Roberto Miranda, Mara Aznar, Jos L. Carrascosa, Iwan A. T. Schaap, David Reguera, and Pedro J. de Pablo.

Direct measurement of phage phi29 stiffness provides evidence of internal pressure.

Small, 8(15):2365-2365, 2012.

[11] Jacob N. Israelachvili.

Intermolecular and Surface Forces.

Elsevier, 3rd edition, 2011.

[12] N. Kikuchi and J. T. Oden.

Contact Problems in Elasticity: A Study of Variational Inequalities and Finite Element Methods.

SIAM, Studies in Applied and Numerical Mathematics, 1988.

[13] Young W. Kwon and Hyochoong Bang.

The Finite Element Method Using MATLAB.

CRC Press, Inc., Boca Raton, FL, USA, 2nd edition, 2000.

[14] J.-L. Lions and G. Stampacchia.

Variational inequalities.

Comm. Pure Appl. Math., 20:493-519, 1967. 
[15] M Marsh and A Helenius.

Virus entry: Open sesame.

Cell, 124(4):729-740, 2006.

[16] Mauricio G Mateu.

Structure and Physics of Viruses, volume 68 of An Integrated Textbook.

Springer Science $\{\&\}$ Business Media, Dordrecht, jun 2013.

[17] J. P. Michel, I. L. Ivanovska, M. M. Gibbons, W. S. Klug, C. M. Knobler, G. J. L. Wuite, and C. F. Schmidt.

Nanoindentation studies of full and empty viral capsids and the effects of capsid protein mutations on elasticity and strength.

Proceedings of the National Academy of Sciences, 103(16):6184-6189, 2006.

[18] W. H. Roos, R. Bruinsma, and G. J. L. Wuite.

Physical virology.

Nature Physics, 6(10):733-743, oct 2010.

[19] W H Roos, M M Gibbons, A Arkhipov, C Uetrecht, N R Watts, P T Wingfield, A C Steven, A J R Heck, K Schulten, W S Klug, G J L Wuite, K Schulte, W S Klug, and G J L Wuite.

Squeezing protein shells: how continuum elastic models, molecular dynamics simulations, and experiments coalesce at the nanoscale.

Biophysical Journal, 99(4):1175-1181, aug 2010.

[20] WouterH. Roos.

How to perform a nanoindentation experiment on a virus.

In Erwin J. G. Peterman and Gijs J. L. Wuite, editors, Single Molecule Analysis, volume 783 of Methods in Molecular Biology, pages 251-264. Humana Press, 2011.

[21] Guido Stampacchia.

Formes bilinéaires coercitives sur les ensembles convexes.

C. R. Acad. Sci. Paris, 258:4413-4416, 1964.

[22] Roger Temam and Alain Miranville.

Mathematical modeling in continuum mechanics.

Cambridge University Press, Cambridge, second edition, 2005.

[23] Reidun Twarock.

Mathematical virology: a novel approach to the structure and assembly of viruses.

Philosophical Transactions of the Royal Society of London A: Mathematical, Physical and Engineering Sciences, 364(1849):3357-3373, 2006.

[24] Robert Vaughan, Brady Tragesser, Peng Ni, Xiang Ma, Bogdan Dragnea, and C. Cheng Kao.

The tripartite virions of the brome mosaic virus have distinct physical properties that affect the timing of the infection process.

Journal of Virology, 88(11):64836491, 2014. 
[25] X. Zeng, S. Mukhopadhyay, and C. L. Brooks.

Residue-level resolution of alphavirus envelope protein interactions in ph-dependent fusion.

Proc. Natl. Acad. Sci. U. S. A., 112(7):2034-2039, 2015. 